\title{
VECTOR DISTANCIA MÍNIMA. UNA NUEVA APROXIMACIÓN PARA EVITAR EL VOLCADO EN MANIPULADORES MÓVILES
}

\section{MINIMAL DISTANCE VECTOR. A NEW APPROACH TO AVOID TIP-OVER ON MOBILE MANIPULATORS}

\author{
Tito Luis González-Fernández \\ Ing. Electrónico \\ Universidad Nacional Experimental del Táchira \\ San Cristobal, Venezuela \\ zulaco64@gmail.com
}

\author{
Antonio José Bravo-Valero \\ Ph. D. Ingeniería \\ Universidad Nacional Experimental del Táchira \\ San Cristobal, Venezuela \\ antonio.j.bravo@gmail.com
}

Resumen- Muchas aplicaciones de los sistemas robóticos requieren que el manipulador desarrolle operaciones mientras se encuentra sobre una plataforma móvil, situación que a diferencia de un manipulador robótico fijo, puede tener un comportamiento inestable y quedar operativamente inutilizado por volcamiento, condición última que puede ser a causa del desplazamiento del centro de gravedad del conjunto al encontrarse sobre el nivel de la plataforma de transporte por efecto del peso del manipulador. Se presenta una nueva técnica para evitar la condición de volcado por la utilización del concepto de distancia mínima entre el centro rotacional del manipulador y el arco proyectado por el centro de masa sobre el suelo de tránsito, con el objeto de modificar el centro de gravedad del conjunto por la rotación del manipulador sobre la plataforma. El objetivo final es compensar el momento rotacional a muy baja velocidad por la utilización del concepto de contra-balance por cambio de posición.

Palabras clave- Centro de gravedad, estabilidad en robots móviles, prevención de volcado, volcamiento.

Abstract- Many future applications of robotics systems will require that manipulators perform operations while being carried by moving vehicles. However, different from a manipulator fixed on the floor, such a vehiclemounted mobile manipulator might be unstable or even tip over, this condition may be because the center of mass is over the mobile platform by the manipulator's weight. This paper presents a new technique to avoid tip-over condition using the concept of minimal distance between the manipulator rotational center and the projection mass center arc in the soil with the object of modificate the gravity center of the set changing the manipulator rotational position on mobile platform, the final objective is to compesate the rotational moment at low velocity using counter-balance concept.

Keywords- Gravity center, robot stability, tipover, turnover prevention.

\section{INTRODUCCIÓN}

Los manipuladores robóticos han incrementado su utilización en aplicaciones en las que no se encuentran unidos al suelo, en su lugar, se han montado sobre plataformas móviles, lo cual ha incrementado ampliamente su rango de acción al operar sobre entornos no estructurados, sin embargo, a diferencia de un manipulador de base fija, la interacción entre el manipulador y el vehículo tiene efecto directo en la estabilidad del conjunto por el problema del volcado, el cual es un serio inconveniente, tanto desde el punto funcional por la anulación operativa del dispositivo como desde el punto de vista de control del robot.

En cuanto al volcado del robot, evitar esta condición requiere de medidas adicionales de la estabilidad, así como de algoritmos de control cuando el dispositivo móvil es teleoperado o transita de manera autónoma, que es el caso más deseado en la operación del robot. En consecuencia se encuentra que mientras los vehículos con orugas y los manipuladores en plataformas móviles por ruedas han sido extensamente estudiados, solo pocos trabajos han sido reportados sobre manipuladores móviles con sistemas de tracción por orugas [1], y muchos menos relacionados con el efecto de la reconfiguración posicional del manipulador sobre la estabilidad de la plataforma móvil o del conjunto.

Este trabajo propone la técnica: Vector Distancia Mínima (VDM) para determinar la posición rotacional del manipulador sobre la plataforma 
móvil que modifique el centro de gravedad del conjunto con la premisa de mejorar la estabilidad del robot.

En este artículo, en la sección primera se realiza la revisión de los trabajos previos en cuanto a la determinación del grado de estabilidad del conjunto. En la sección segunda se trabaja con el modelado matemático de la proyección del centro de gravedad. En la sección tercera se realiza el desarrollo de la propuesta de vector distancia mínima. En la sección quinta se presentan las conclusiones.

\subsection{Revisión bibliográfica}

De entre los trabajos relacionados con el problema del volcado, se pueden destacar los aportes de:

Huang, Q. et al. [2] establecen el concepto de Zero Moment Point (ZMP) y definen el grado de estabilidad como la medida cuantitativa de la extensión estable del manipulador en concordancia con la relación entre la posición del ZMP y la región estable. Papadopoulos, E. et al. [3] implantan la importancia del centro de gravedad en el concepto de estabilidad del robot, ya que la proyección de este dentro del polígono de soporte determina el margen de estabilidad instantáneo del conjunto. Torige, A. et al. [4] trabajan con una plataforma móvil que es más pequeña que el manipulador y, por tanto, la posición del centro de gravedad es controlada por la posición del manipulador, proponiendo un método simple de proyección gráfica para compensar la fuerza de inercia a causa de la aceleración del móvil.

Rey, D.A. et al. [5] describen los dos tipos de inestabilidad de volcado que pueden ocurrir en manipuladores móviles operando en terrenos disparejos; volcado por inestabilidad estática, y volcado por inestabilidad dinámica. Se utiliza la medición de fuerzas-ángulos estáticos y dinámicos para desarrollar el esquema de control. DiazCalderón Antonio, et al. [6] enuncian la utilidad del concepto de polígono soporte o estabilidad, formado por los puntos de contacto del robot con el suelo, siempre y cuando las fuerzas inerciales sean de magnitud pequeña de tal forma que sea factible ignorar su efecto. Nakamura, S. et al. [7] se plantea un robot explorador que puede modificar su centro de masa para transitar sobre terre- nos ásperos o accidentados, en concordancia con sus ángulos de inclinación y utilizando métodos de cálculo en línea para determinar la posición óptima de su centro de masa.

Liu Yugang et al. [8] demuestran que la influencia del manipulador sobre el móvil no puede ser ignorada porque las fuerzas centrifugas experimentadas por el manipulador no se distribuyen como una carga uniforme sobre las cadenas de tracción. Zhu Mingchao et al. [9] utilizan lógica difusa para modelar la dinámica desconocida de manipuladores reconfigurables como subsistemas interconectados, estableciendo la importancia del control descentralizado por la reducción en la complejidad del esquema de control y los costos de cálculo.

Morales, J. et al. [10] realizan el estudio experimental sobre el efecto que tiene la reubicación del manipulador de elevado peso, sobre el desplazamiento del centro de gravedad del conjunto y su efecto sobre la estabilidad general del sistema. Roan, P.R. et al. [11] validan con información experimental tres algoritmos para evitar la condición de volcado en un manipulador móvil. El pequeño número de algoritmos utilizados es debido al gran costo de cálculo en la implementación de las muchas propuestas que se han dado a conocer. Se indica que el factor primordial en la condición de volcado es cuando se transportan cargas que elevan el centro de gravedad del conjunto.

\section{MODELADO MATEMÁTICO DE LA PROYECCIÓN DEL CENTRO DE GRAVEDAD}

En cuanto al modelado del robot se utilizaron las expresiones matemáticas establecidas por Morales J., et al. [10], que son indicadas en (1), (2), (3), (4), (5), (6) y (7) teniendo por objeto representar el comportamiento de la proyección del centro de gravedad sobre la horizontal en función de: $\alpha$, ángulo de inclinación longitudinal. $\beta$, ángulo de inclinación transversal. $\theta$, ángulo de rotación del manipulador.

$$
\begin{gathered}
x_{g}=R \cdot \cos (1.016 \cdot \theta+87.33)+x_{c} \\
y_{g}=R \cdot \operatorname{sen}(1.016 \cdot \theta+87.33)+y_{c} \\
x_{p r o j}=x_{g}+\tan (\beta) \cdot z_{g}
\end{gathered}
$$




$$
\begin{gathered}
y_{p r o j}=y_{g}-\tan (\alpha) \cdot z_{g} \\
d_{x}=\frac{w}{2}-\left|x_{p r o j}\right| \\
d_{y}=\frac{1}{2}-\left|y_{p r o j}\right| \\
d=\max \left(\min \left(d_{x}, d_{y}\right)\right)
\end{gathered}
$$

Donde las variables se encuentran identificadas de la siguiente manera: $x_{g}, y_{g}$ son las coordenadas $\mathrm{X}, \mathrm{Y}$ del centro de gravedad proyectado sobre la horizontal absoluta $\left(\alpha=\beta=0^{\circ}\right)$. $R$ es el radio de rotación del manipulador. $x_{c}, y_{c}$ son las coordenadas $X, Y$ del eje o centro de rotación del manipulador. $x_{\text {proj }}, y_{\text {proj }}$ son las coordenadas $X, Y$ del centro de gravedad proyectado en función de la inclinación frontal, lateral, y la altura del centro de gravedad. $z_{g}$, es la altura sobre la plataforma del centro de gravedad. $d x$ y $d y$, son las distancias de la proyección del centro de gravedad por la rotación del manipulador sobre la plataforma móvil a los límites del polígono de estabilidad. $w$ es el ancho del polígono de estabilidad. I es el lar- go del polígono de estabilidad. $d$ es el índice de estabilidad estática general y es el mayor valor de los menores valores resultantes de la intersección entre $d x$ y $d y$.

\subsection{Primera herramienta de simulación numérica}

Desde (1) hasta (7) fueron codificadas en un archivo tipo guión (script) para ser interpretadas por el software MATLAB, de manera tal que efectuando la combinación estructurada de distintos valores numéricos en los ángulos $\alpha$ y $\beta$, en combinación con el barrido del ángulo $\theta$ desde su valor mínimo hasta su valor máximo fue posible apreciar la proyección del arco del centro de gravedad en función de estos ángulos. La herramienta desarrollada después de realizar los cálculos correspondientes genera una figura compuesta por 4 subfiguras, las cuales representan de manera simplificada el cuerpo del robot, su proyección del centro de gravedad al rotar el manipulador, los valores de los vectores de los índices de estabilidad $d x$ y $d y$, el vector $d$ y el marcador del ángulo $\theta$ con el mayor índice de estabilidad, como se muestra en la Fig. 1.

Fig. 1. PRIMERA HERRAMIENTA DE SIMULACIÓN NUMÉRICA
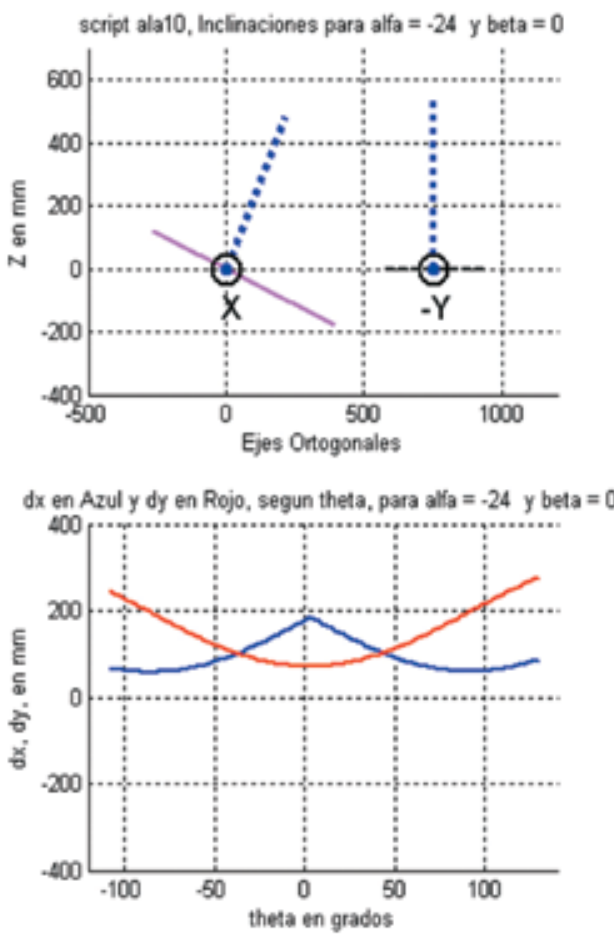
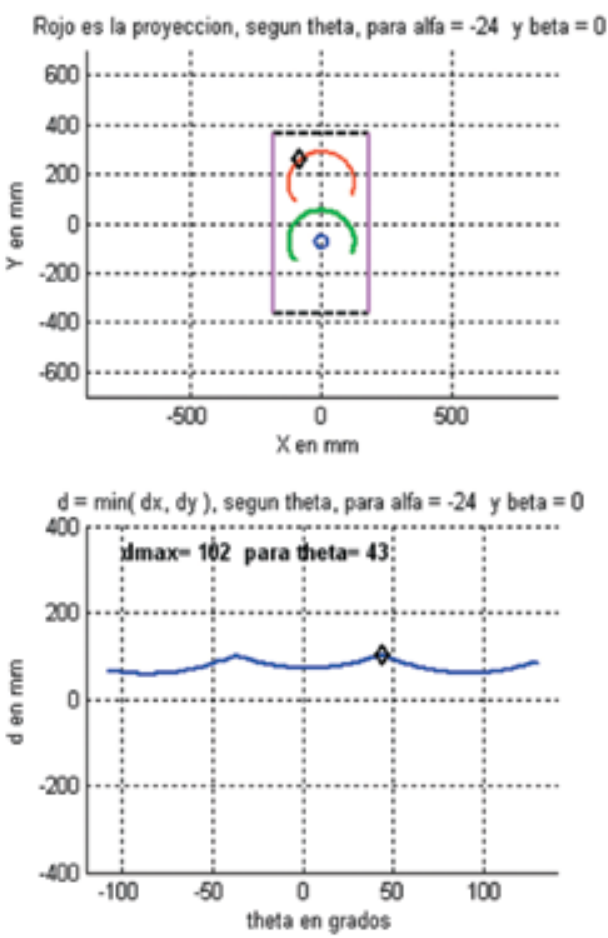


\subsection{Resultados de la primera herramienta de simulación numérica}

De los resultados obtenidos y visualizados por la combinación de distintos valores de los ángulos $\alpha$ y $\beta$, se hace necesario resaltar que en relación con las inclinaciones frontales en las cuales el ángulo $\alpha$ varíe desde $-30^{\circ}$ hasta -15 grados, el conjunto de valores de la componente $d x$ describe una curva convexa mientras que el conjunto de valores de la componente dy describe una curva que es cóncava con lo que al interceptarse para determinar los valores mínimos, se producen dos valores máximos, dentro del conjunto de mínimos, que se encuentran reflejados con respecto al eje $X=0$ y numéricamente muy próximos entre sí de manera tal que al ser determinado el valor del índice de estabilidad se presenta una alternancia que en función del ángulo $\alpha$ determina oscilaciones acentuadas para el ángulo $\theta$, con alternancias de signo, ya que va de valores de ángulo positivos a valores de ángulo negativos, y viceversa, de forma tal que al ser utilizados como referencia en el controlador de posición del manipulador anulan la obtención de un punto de equilibrio, Fig. 1.

Una comprobación de lo anteriormente indicado se puede apreciar en la Fig. 2, en la cual se grafica por medio de un guión particular de MATLAB los resultados para una inclinación simple derecha-izquierda en color azul y con incremento angular de 5 grados en $\beta$, y una inclinación simple frente-atrás en color rojo y con incremento angular de 2 grados en $\alpha$, donde se observa perfectamente el problema de las oscilaciones en el valor de referencia para el controlador del manipulador móvil.

Fig. 2. ZONA DE OSCILACIÓN DEL ÍNDICE DE ESTABILIDAD d

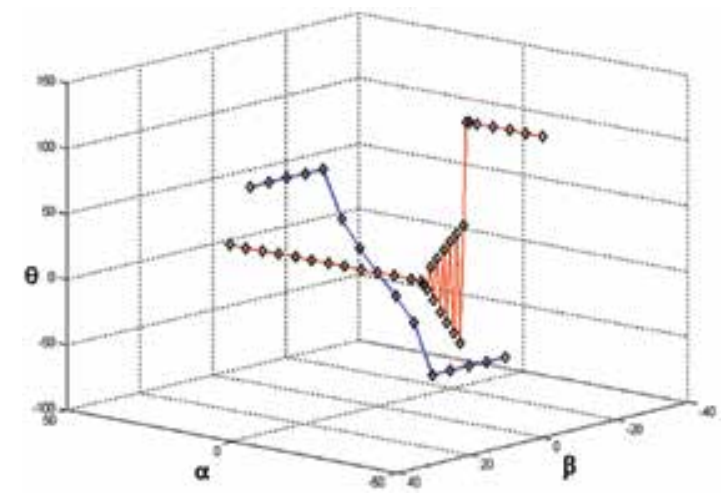

Fuente: autores.
En lo referente a las inclinaciones frontales, se determina que por efecto de la diversidad de condiciones que se presentan para determinar el valor del índice de estabilidad en función de las componentes $d x$ y dy debido a su dependencia de los rangos de valores del ángulo $\alpha$, el método de determinación de este índice por medio de (5), (6) y (7) se pone en duda, ya que no es el mejor indicador para realizar un algoritmo de control, porque presenta puntos de incongruencia o puntos de ambigüedad para tomar la decisión que determine la acción de control para ejecutar.

De los resultados anteriores se infiere que pueden existir otras zonas angulares de $\alpha, \beta$, o combinación de estos ángulos tanto para movimiento lineal como para un movimiento mixto o diagonal en los cuales se presente este problema de oscilaciones, aparte de la condición operativa del valor de incremento de los ángulos a efecto de simulación numérica.

En esa misma idea y utilizando como software de simulación numérica a MATLAB, se desarrolló el script que al ser ejecutado realice un barrido combinatorio entre los ángulos $\alpha$ y $\beta$ para que con un incremento angular de $1^{\circ}$ se determine de manera puntual en cada combinación el valor del ángulo $\theta$ que corresponde con el índice de estabilidad, de esta manera se espera lograr una superficie en la que pueda ser posible detectar las irregularidades que indiquen la posibilidad de oscilaciones en el índice de estabilidad y por ende en el valor de $\theta$ que le corresponde.

En la Fig. 3 se puede observar la superficie obtenida según lo establecido previamente, indicándose las posibles zonas de oscilación. Otro aspecto que se puede observar es la existencia de varias zonas de transición o cambio brusco en el valor del ángulo $\theta$, lo cual ratifica que es un método inadecuado para determinar la referencia, utilizado por el controlador de posición del manipulador al rotar este sobre la plataforma móvil.

Habiendo quedado claro el hecho de que el método establecido por (7) no es el idóneo para determinar el índice de estabilidad $d$ en función de las componentes $d x$, por (5), y $d y$, por (6), de la proyección del centro de gravedad en función del ángulo $\theta$, se hace necesario formular la siguiente pregunta: ¿Que método o técnica utilizar para determinar el valor del índice de estabilidad cumpliendo con el criterio de proyección del centro de gravedad? 
Fig. 3. SUPERFICIE DEL ÍNDICE DE ESTABILIDAD SEGÚN LOS VALORES $D E \alpha Y \beta$

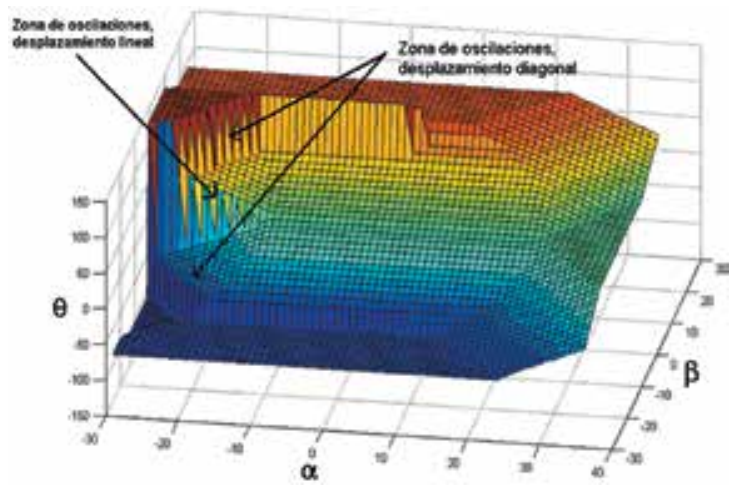

Fuente: autores.

\section{EQUILIBRIO DE MOMENTOS, VECTOR DISTANCIA MÍNIMA}

En relación con la pregunta antes planteada, varios de los artículos revisados manejan el aspecto de la cinemática de un manipulador móvil por medio de modelado matemático que requiere de ecuaciones de elevada complejidad, sin embargo, la comprobación experimental es sobre terreno horizontal y sin irregularidades, ya que los costos de cálculo a nivel de una plataforma móvil y autónoma son muy elevados para la aplicación.

En función de lo establecido previamente se infiere que hay la necesidad de desarrollar un método que sea de bajo costo computacional, reactivo de ser posible, para que pueda ser utilizado a efectos de control del centro de gravedad del manipulador móvil o por cualquier otro tipo de configuración robótica.

Como se ha establecido en los artículos revisados, a baja velocidad la estabilidad del robot es principalmente función de la posición del centro de gravedad, sobre todo, si este se encuentra elevado o fuera del cuerpo del robot, condición que tiene relación directa con el concepto del momento angular o momento cinético, el cual es una magnitud física importante en todas las teorías físicas de la mecánica, desde la clásica o Newtoniana a la cuántica, pasando por la mecánica relativista.

Su importancia en todas ellas se debe a que está relacionada con las simetrías rotacionales de los sistemas físicos, en los que bajo ciertas condiciones es una magnitud que se mantiene constante con el tiempo a medida que el sistema evo- luciona, lo cual da lugar a una ley de conservación conocida como ley de conservación del momento angular, la cual se enuncia en mecánica newtoniana, como:

$$
\sum_{i=1}^{n} \vec{F}_{i} \times r_{i}=0
$$

siendo $\overrightarrow{\mathrm{F}}_{i}$ los vectores de fuerzas aplicadas al objeto, y $r_{i}$ los brazos de palanca de cada fuerza aplicada.

En (8) se establece que para poder equilibrar un sistema cualquiera desde el punto de vista estático es necesario utilizar un contrapeso, lo cual indica que la ubicación de esta masa de compensación debe ser diametralmente opuesta al cuerpo que causa el desequilibrio, o la rotación en la ubicación del testigo de manera diametralmente opuesta a la inclinación que causa el desbalance.

Para la configuración de manipulador móvil en la cual el centro de masa se ubique elevado de la plataforma móvil, al realizar un diagrama de cuerpo libre, Fig. 4, se aprecia que si bien no hay un contrapeso como tal para lograr la condición de equilibrio, se puede observar que la fuerza de reacción (R) que se produce en los puntos de apoyo del sistema de tracción, al descomponerla en sus componentes en el centro de gravedad, es la que genera el efecto de compensación que garantiza la condición de equilibrio, y que se manifiesta en la proyección del centro de gravedad sobre el polígono de estabilidad [10].

Fig. 4. DIAGRAMA DE CUERPO LIBRE

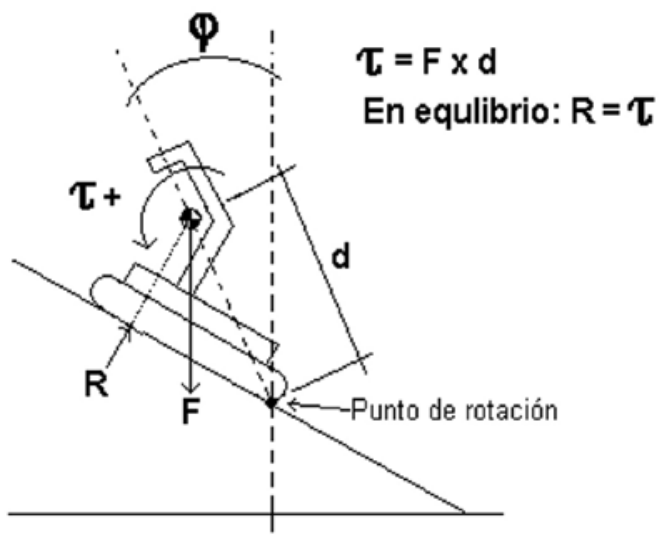

Fuente: autores.

En (9) se indica la condición de equilibrio para este sistema. 


$$
R=\tau=F \times d
$$

Si el terreno es suficientemente resistente a la deformación, se puede establecer que la reacción siempre compensará el momento del conjunto, ya que es exactamente igual a este, condición a su vez en la que al realizar una comparación del efecto de la rotación del manipulador para una inclinación constante, permite inferir la posibilidad de utilizar al ángulo $\varphi$ como un indicador de la estabilidad del conjunto, ya que a mayor valor, mayor se puede considerar la estabilidad estática del sistema como se indica en la Fig. 5.

Sin embargo, la medición de este ángulo es complicada, ya que debe hacerse de manera indirecta aparte de que presenta el inconveniente de las componentes por el valor de rotación en el ángulo $\theta$. En cualquier caso, es obvio el beneficio de rotar el manipulador hasta una posición diametralmente opuesta a la inclinación a que se encuentra sometido el robot, esto a manera de contrapeso para lograr el balance de momentos. En este punto, se hace necesario indicar que para el caso particular del robot ALACRANE este no puede rotar el manipulador hasta los $180^{\circ}$ por efecto de los topes mecánicos al encontrarse configurado el mismo en la posición de avance horizontal [10].
Fig. 5. EFECTO DE ROTACIÓN DEL MANIPULADOR

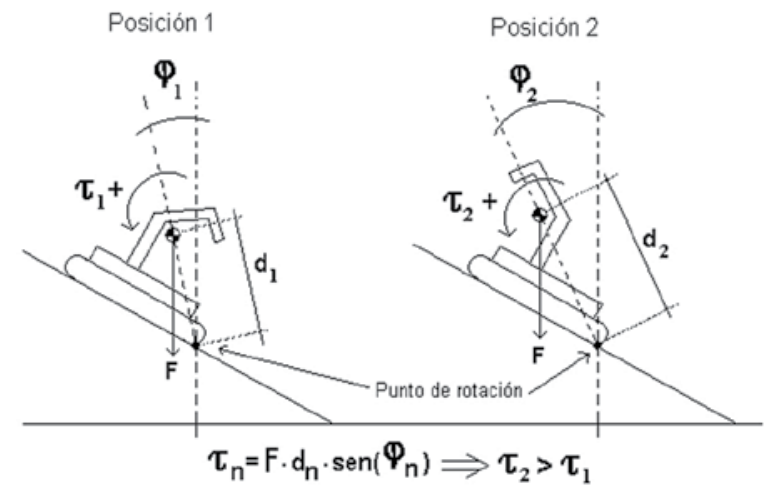

Fuente: autores.

En relación con la Fig. 5, en (10) se establecen las consideraciones de estabilidad en función de cada posición según del ángulo $\varphi$ correspondiente.

$$
\tau_{n}=F \cdot d_{n} \cdot \operatorname{sen}\left(\varphi_{n}\right) \Rightarrow \tau_{2}>\tau_{1}
$$

Por otra parte, trabajando con las proyecciones del centro de gravedad del manipulador en función del ángulo $\theta$ para inclinaciones laterales simples con valores de ángulo extremo, Fig. 6(a) para inclinación a la izquierda, y Fig. 6(b) para inclinación a la derecha, se observa que si se toma como referencia las coordenadas del eje de rotación del manipulador, y luego se mide la distancia a cada

Fig. 6. VECTOR DISTANCIA MÍNIMA PARA INCLINACIONES LATERALES SIMPLES
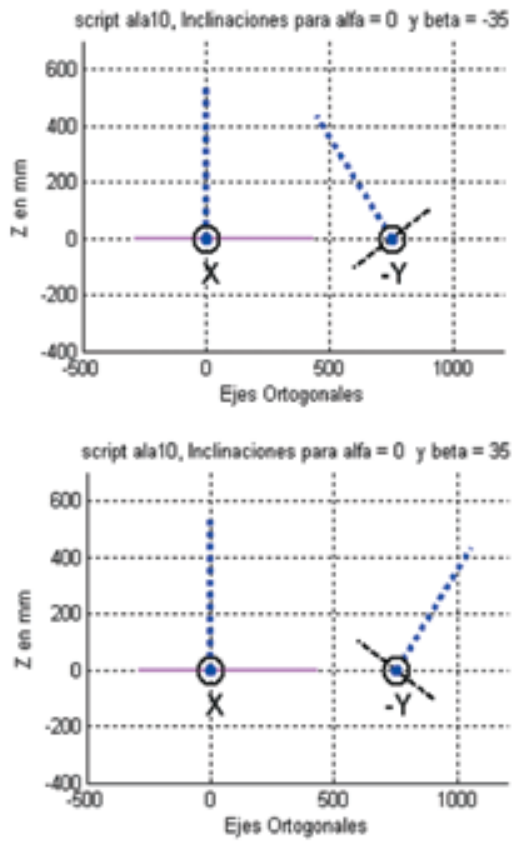
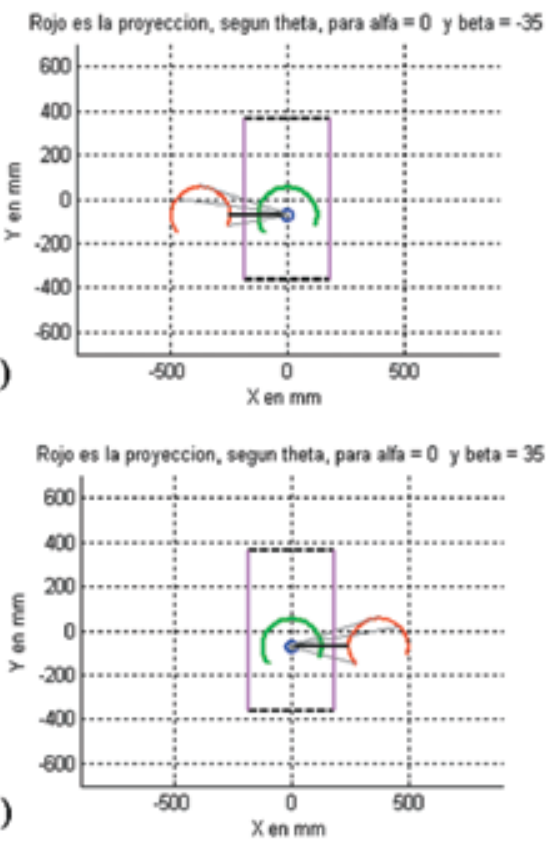
uno de los puntos de la proyección del centro de gravedad en función de $\theta$, líneas de color gris, es posible determinar cuál es el vector distancia mínima, línea de color negro, de la que se determina a su vez que corresponde con el valor del ángulo $\theta$ para el cual se obtiene la compensación necesaria por contrapeso según el concepto de balance de momentos.

Si ahora se aplica el concepto VDM a las proyecciones del centro de gravedad del manipulador en función del ángulo $\theta$ para inclinaciones frontales simples con valores de ángulo extremo, Fig. 7 , se aprecia que para el caso de inclinación hacia el frente, Fig. 7(a) con $\alpha$ menor que $0^{\circ}$, existe la posibilidad de dos soluciones si el valor de los ángulos de $\theta$ para tope a derechas y tope a izquierdas fueren iguales, condición que no se presenta en el robot ALACRANE, ya que el tope a derechas se da para el ángulo $\theta=+130^{\circ}$ y el tope a izquierdas se da para el ángulo $\theta=-108^{\circ}$ [10]. En cualquier caso, se aprecia que la distancia mínima para una inclinación hacia el frente es para un valor del ángulo $\theta$ que indica una rotación hacia atrás, lo cual nos suministra compensación por contrapeso como ya fue establecido previamente.

Observación particular merece la Fig. 7(b), en la que el sistema se encuentra ahora inclinado ha- cia atrás, es decir, el valor del ángulo $\alpha$ es mayor que $0^{\circ}$, con lo cual el vector de distancia mínima corresponde a un valor del ángulo $\theta$ de $2^{\circ}$, con lo cual puede afirmarse que si la inclinación es hacia atrás, la compensación por balanceo de momentos es rotando al manipulador hacia el frente, a la posición de avance [10]. En (11) se aprecia la expresión que determina la menor distancia.

$$
d_{m}=\sqrt{\left(x_{2}-x_{1}\right)^{2}+\left(y_{2}-y_{1}\right)^{2}}
$$

En relación con el concepto de distancia mínima, el punto de referencia se ha establecido en las coordenadas del eje de rotación del manipulador en la plataforma móvil, sin embargo, nada impide que se utilice cualquier otro punto de referencia para el cálculo de la distancia hasta la proyección del centro de gravedad en función de $\theta$, siempre y cuando el mismo sea un valor común a todas las mediciones, incluso este punto de referencia puede estar fuera de las coordenadas de la plataforma móvil, fuera del robot.

Si bien, en ese sentido se hubiese podido seleccionar las coordenadas origen $(0,0)$ de la plataforma móvil, para los resultados que se presentarán de ahora en adelante se selecciona el eje de rotación del manipulador por su misma connotación de eje.

Fig. 7. VECTOR DISTANCIA MÍNIMA PARA INCLINACIONES FRONTALES SIMPLES
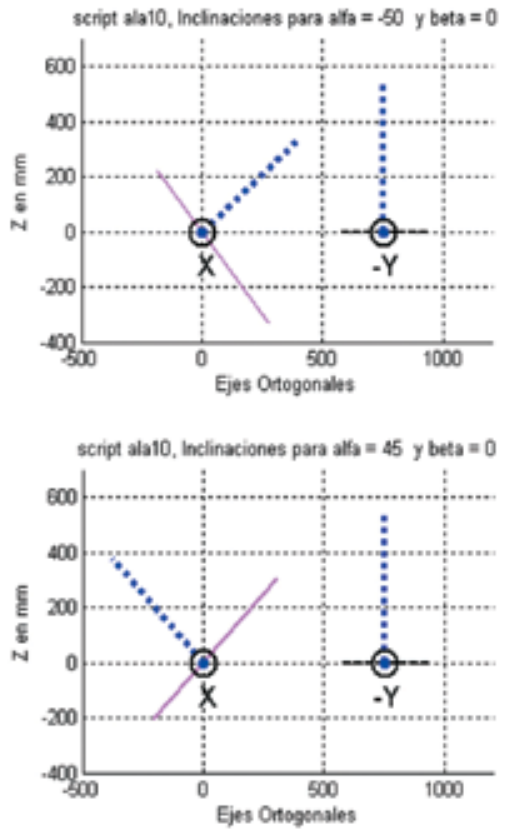

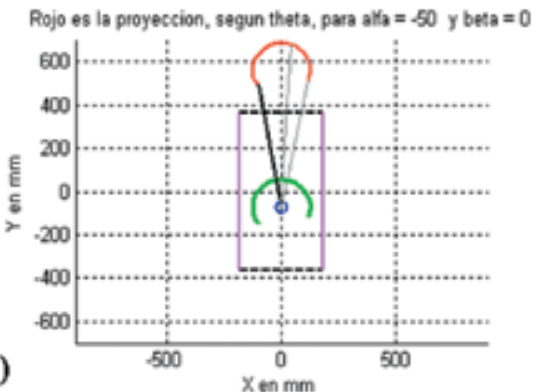

(b)

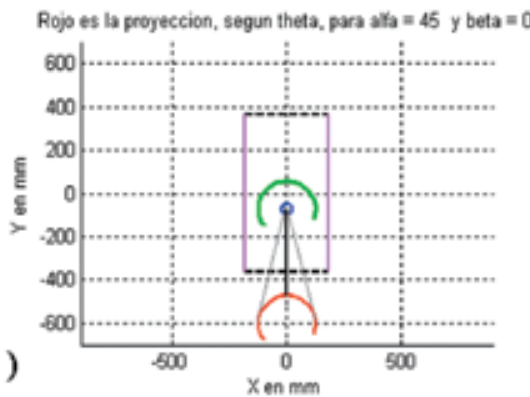


Caso particular será cuando el robot se encuentre sobre la horizontal absoluta, $\alpha=\beta=0^{\circ}$, ya que en ese caso la distancia del eje de rotación a la proyección del centro de gravedad será la misma para cualquier valor del ángulo $\theta$, en ese caso debe crearse una condición especial en el programa de simulación para tomar la posición de avance, puesto que es la única inclinación para la cual ocurre esta condición.

Si bien, conceptualmente la distancia mínima parece ser válida, se hace necesario realizar una serie de pruebas que permitan una comprobación simple del concepto, para poder ser utilizado en los pasos siguientes.

\subsection{Segunda herramienta de simulación numérica}

Como se demuestra la inconveniencia de la utilización del índice de estabilidad d, definido en (5), el cual es sustituido por el vector distancia mínima, definido en (11), se genera un nuevo guión de MATLAB para simulación y en el cual se representa de manera simplificada el cuerpo del robot, la proyección del centro de gravedad al rotar el manipulador, los valores del vector de distancias en el que se aprecia el marcador del ángulo $\theta$ para el cual se obtiene la mínima distancia al eje de rotación del manipulador. Como se muestra en la Fig. 8.

Fig. 8. SEGUNDA HERRAMIENTA DE SIMULACIÓN NUMÉRICA
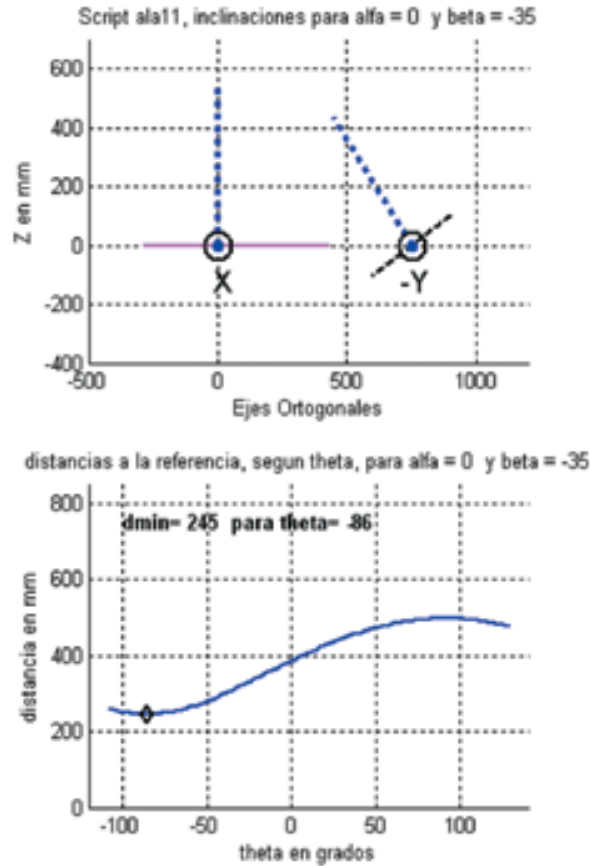

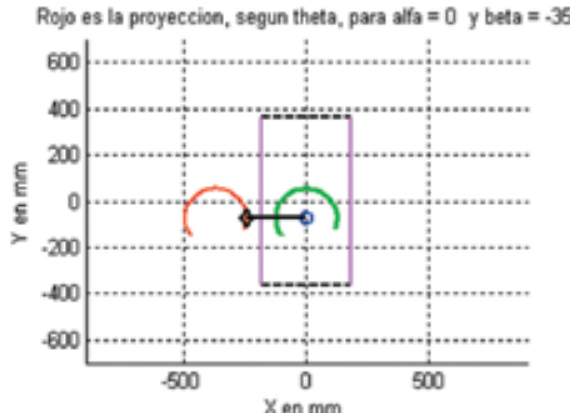

Fuente: autores.

\subsection{Resultados de la segunda herramienta de simulación numérica}

De las simulaciones de desplazamientos simples de inclinación frontal se infiere que el concepto de distancia mínima es correcto para lograr el equilibrio o balanceo de momentos, ya que no existe el inconveniente de oscilaciones en la posición que debe tomar el manipulador por la rotación sobre su eje en la plataforma móvil, para lograr la condición de balance.
Una comprobación de lo antes indicado se puede apreciar en la Fig. 9, en la cual se grafica por medio de un guión particular de MATLAB los resultados para una inclinación simple derechaizquierda en color azul y con incremento angular de 5 grados en $\beta$, y una inclinación simple frenteatrás en color rojo y con incremento angular de 2 grados en $\alpha$, donde se observa perfectamente que los marcadores no muestran el problema de las oscilaciones en el valor de referencia para el controlador del manipulador móvil, en la zona que 
anteriormente oscilaba con el incremento como se muestra en la Fig. 2.

Fig. 9. VISUALIZACIÓN DE LA ZONA DE OSCILACIÓN UTILIZANDO EL VECTOR DE DISTANCIA MÍNIMA

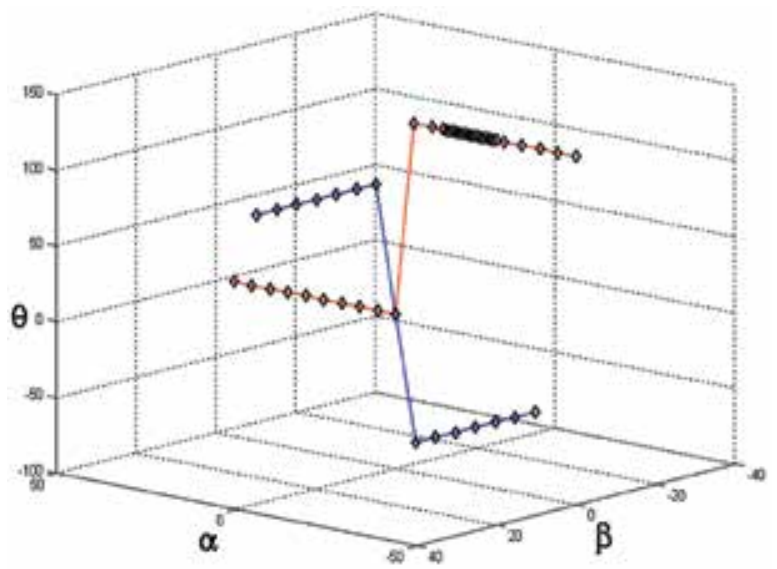

Fuente: autores.

Si bien los resultados observados en la Fig. 9 indican que el concepto del vector de distancia mínima funciona adecuadamente por su relación directa con el concepto de balaceo de momentos, es obvio que no se han realizado todas las combinaciones posibles. En tal sentido, y al igual que para la Fig. 3, se determina la superficie que describe el comportamiento del marcador de distancia mínima en función del valor de los ángulos $\alpha$ y $\beta$.

De la Fig. 10 se aprecia que el concepto de vector de distancia mínima es válido para la determinación del marcador de punto de balance, ya que no se observan discontinuidades que indiquen zonas de oscilación, por el contrario, se observan zonas angulares de cambio gradual o suave cuando el valor del ángulo $\alpha$ es mayor que $0^{\circ}$, mientras que para el caso de inclinación hacia el frente cuando el valor del ángulo $\alpha$ es menor que $0^{\circ}$, hay una sola transición que es la que lleva la posición del manipulador de un extremo hacia el otro, lo cual implica el recorrido completo del arco de rotación. Además, también se aprecia que hay zonas donde el valor del ángulo $\theta$ permanece constante.

Por otra parte, la validez de la superficie mostrada en la Fig. 10 fue comprobada a nivel de simulación, por su utilización en el desarrollo de un controlador en lógica difusa para la reubicación del manipulador sobre la plataforma móvil [12].
Fig. 10. SUPERFICIE DEL VECTOR DE DISTANCIA MÍNIMA SEGÚN VALOR DE ÁNGULOS $\alpha, \beta$, y $\theta$

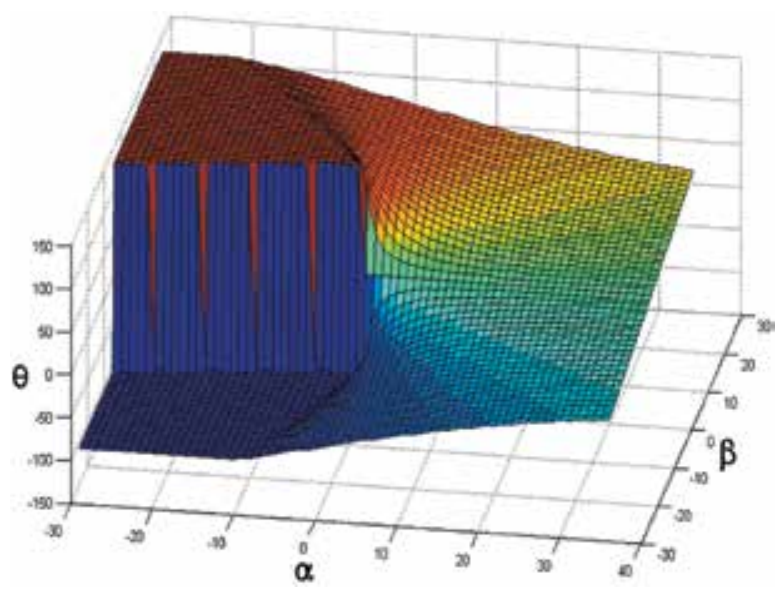

Fuente: autores.

\section{CONCLUSIONES}

Se determina que la técnica del valor del índice de estabilidad $d$, descrita en (5) por las componentes $d x$, dy según la proyección del centro de gravedad en función del valor de los ángulos $\alpha, \beta$, y $\theta$ sobre el polígono de estabilidad, no es la más adecuada para realizar un algoritmo de control, ya que presenta puntos de ambigüedad para indicar la referencia que debe seguir la acción de control por ejecutar.

Se demuestra que la técnica del vector de distancia mínima descrita en (11) es la más apropiada para indicar la referencia que debe seguir la acción de control por ejecutar, ya que se encuentra en concordancia con el concepto de balance de momentos para lograr una posición de equilibrio estático, lo que permite un comportamiento reactivo en las acciones de control, con lo cual se disminuyen ampliamente los costos computacionales.

\section{REFERENCIAS}

[1] Y. Liu, G. Liu, "Modeling of tracked mobile manipulators with consideration of track-terrain and vehiclemanipulator interactions", Robotics and Autonomous Systems, vol. 57, no. 11, pp. 1065-1074, Nov 2009.

[2] Q. Huang, S. Sugano, I. Kato, "Stability control for a mobile manipulator using a potential method", Intelligent Robots and Systems, vol. 2, no. 1, pp. 839-846, Apr. 1994. 
[3] D. A. Rey, E. G. Papadoupoulos, "A new measure of tipover stability margin for Mobile manipulators", Robotics and Automation, vol. 4, no. 1, pp. 3111-3116, May. 1996.

[4] A. Torige, T. Ihara, "Control of manipulator with gravity center position control on Mobile vehicle", Advanced Robotics, vol. 1, no. 1, pp. 367-372, Jun. 1997.

[5] D. A. Rey, E. G. Papadoupoulos, "Online automatic tipover prevention for mobile manipulators", Intelligent Robots and Systems, vol. 3, no. 1, pp. 1273-1278, Dec. 1997.

[6] A. Díaz-Calderon, A. Kelly, "On the dynamic stability of mobile manipulators", PhD Thesis, the Robotics Institute, Carnegie Mellon University. Pittsburgh, PA, USA, 2000.

[7] S. Nakamura, M. Faragalli, N. Mizukami, et al., "Wheeled robot with movable center of mass for traversing over rough terrain", Intelligent Robots and Systems, vol. 2, no. 1, pp. 1228-1233, Jan. 2007.
[8] L. Yugang, L. Guangjun, "Kinematics and interaction analysis for tracked mobile manipulators", Intelligent Robots and Systems, vol. 1, no. 1, pp. 267-272, Jan. 2007.

[9] Z. Mingchao, L. Yuanchung, "Decentralized adaptive fuzzy control for reconfigurable manipulators", Robotics, Automation and Mechatronics, vol. 1, no. 1, pp. 404-409, Feb. 2008.

[10] J. Morales, J. Martínez, A. Mandow, et al, "Center of gravity estimation and control for a field mobile robot with a Heavy Manipulator", Mechatronics, vol. 1, no. 1, pp. 1-6, Mar. 2009.

[11] P. R. Roan, A. Burmeister, A. Rahimi, et al, "Real-world validation of three tipover algorithms for mobile robots ", Robotics and Automation, vol. 5, no. 1, pp. 44314436, Aug. 2010.

[12] T. González, A. Bravo, "Controlador Difuso para el Cambio del Centro de Gravedad en Manipuladores Móviles”, IEEE América Latina, vol. 12, no. 6, pp. 991996, Sept. 2014. 